\title{
Role of plasticity-induced crack closure in fatigue crack growth
}

\author{
Jesús Toribio, Viktor Kharin \\ University of Salamanca, Spain
}

\begin{abstract}
The premature contact of crack surfaces attributable to the near-tip plastic deformations under cyclic loading, which is commonly referred to as plasticity induced crack closure (PICC), has long been focused as supposedly controlling factor of fatigue crack growth (FCG). Nevertheless, when the plane-strain near-tip constraint is approached, PICC lacks of straightforward evidence, so that its significance in FCG, and even the very existence, remain debatable. To add insights into this matter, large-deformation elastoplastic simulations of plane-strain crack under constant amplitude load cycling at different load ranges and ratios, as well as with an overload, have been performed. Modeling visualizes the Laird-Smith conceptual mechanism of FCG by plastic blunting and re-sharpening. Simulation reproduces the experimental trends of FCG concerning the roles of stress intensity factor range and overload, but PICC has never been detected. Near-tip deformation patterns discard the filling-in a crack with material stretched out of the crack plane in the wake behind the tip as supposed PICC origin. Despite the absence of closure, load-deformation curves appear bent, which raises doubts about the trustworthiness of closure assessment from the compliance variation. This demonstrates ambiguities of PICC as a supposedly intrinsic factor of FCG and, by implication, favors the stresses and strains in front of the crack tip as genuine fatigue drivers.
\end{abstract}

KEYwORDS. Crack closure; Crack-tip strains; Fatigue cracking.

\section{INTRODUCTION}

$\mathrm{T}$ he capability of opposite crack surfaces to contact prematurely at unloading due to particular patterns of the neartip plastic straining under cyclic loading, the so called plasticity induced crack closure (PICC), is believed to be the intrinsic feature of fatigue crack growth (FCG), cf., e.g., the Overview [1], although not everybody involved in fatigue studies shares this conviction [2-4]. Despite the phenomenon of crack closure has been focused for decades since its raising by Elber [5], neither agreement between evaluations by different ways nor consensus about the relevance, and even the very existence, of PICC in the course of FCG do exist, cf. [1-3,6,7]. Conclusions about the PICC mechanisms based on both dislocation and continuum plasticity analyses are contradictory, cf., e.g., Deshpande et al. [8] or Pippan and Riemelmoser [9] vs. Louat et al. [3] or Bjerkén and Melin [10], Budianski and Hutchinson [11] vs. Noroozi et al. [12], and McClung et al. [13] vs. Toribio and Kharin [14].

PICC is used to be accepted as the factor directly responsible for the dependence of FCG, apart from the load range, on the load maximum or ratio, as well as on over- or under-loads along the loading routes, forming thus the framework to interpret FCG trends. Crack closure, which can arise from various origins (incidental ones, such as in-crack debris, oxides or other in-crack depositions, as well as ubiquitous ones, such as the crack surface roughness), seems to be out of doubts as a phenomenon that can accompany FCG and affect it under certain circumstances. Nevertheless, specifically PICC remains questionable as the universal intrinsic mechanism that controls FCG. A deal of uncertainty owes here to the difficulties of straightforward evidence of the crack closure phenomenon. Reviews $[1,6,7]$ catalogued a variety of ways of the crack closure verification and assessment ranging from deductions based on the crack growth behavior (i.e., on the presumption of the role of closure in the process) to interpreting the compliance measurements on the assumption that 
the experienced nonlinearity of the load-deformation curves (as obtained with the use of displacement or strain gauges, interferometry, or other devices to monitor deformations) "indicates that there is a progressive change in geometry of the specimen, which must be caused by the closing of the crack" [5]. This way, the very identification of PICC lacks of direct evidence and its responsibilities in FCG remain debatable.

Since in situ visualization of the near-tip deformations is hardly feasible in the places where the plane-strain situation is approached, computational simulation turns out to be the right way to determine them. Accounting for both constitutive (inelasticity) and geometrical (large displacements and strains) nonlinearities is here essential. For cracks growing by means of local material rupture, the alteration of solid boundary via crack advance by bond breaking is the third nonlinearity. Then, the most precise revelation of the near tip situation would be achieved taking into account all three mentioned nonlinearities. Up to date, among available analyses of fatigue cracks, some of them have not accounted for large deformations (such as, e.g., $[13,15,16]$ ), whereas others (e.g., $[14,17-24]$ ), although fulfilling this deficiency, presented partial data about cracks under cyclic loading. Anywise, common shortcoming is the lack of realistic treatment of the crack growth by means of bond breaking. This latter was simulated usually by cutting the material bonds fairly subjectively along finite-size steps via finite element node release to the analyst's discretion at the bottom, mid or top points of designated loading cycles irrespectively of both the material and applied load (cf., e.g., [13, 16, 21, 24]), and thus, having hardly whatsoever to do with the physics of crack advance. The efforts involving more realistic mechanisms of material damage and bond breaking directly in the modeling of FCG have been undertaken since nearly a decade (e.g., [25, 26]) usually by substantial computational expenses. However, these relatively few attempts are at rather early stage of development and encounter conceptual and technical difficulties on the way to implement damage mechanics in large-deformation formulations. Finally, having difficulties with direct involvement of realistic material rupture modeling into simulation of FCG, another approach counts on the stress-, strain- or energy-based variables as fatigue damage monitors [27-30]. However, the evolution of a tip of a growing crack cannot be solved there straightforwardly.

Obviously, ignoring any of the three commented nonlinearities worsens the resolution of the crack-tip fields. Disregard among them of the crack growth via bond breaking, modeling of which is surrounded by the most controversy, may have significance for the simulation outcome. However, would consequent inaccuracy be more or less substantial in comparison with faultiness of unrealistic modeling of the bond breaking merely by making use of a "virtual knife" in analyst's hands has to be verified through much advanced analyses involving duly all three concerned nonlinearities.

Meanwhile, among conceptual mechanisms of FCG, the one, which was suggested by Laird and Smith [31] and reaffirmed by Pelloux [32] and Neumann [33], and which was emphasized as the rational physical model for FCG in ductile materials $[2,10,22,23,34,35]$, relies solely on the near tip plastic deformations under cyclic loading without involvement of pullapart bond breaking. Then, high-resolution large-deformation elastoplastic simulation is the right way to visualize this mode of FCG. Recent advances in large-strain elastoplastic analyses of cracks under cyclic loading [14,17-20,22,23] revealed various aspects of FCG according to the Laird-Smith concept.

The scope of this paper is to narrate the results of modeling of cracks under cyclic loading, which are in particular association with the matter of PICC verification and assessment, and have not been revealed in previous presentations $[14,18-20]$ of the performed simulations. Deformations near the crack subjected to mode I cyclic loading under plane strain and small scale yielding (SSY) are described with the intention to bring insight into some long-standing controversies about the crack blunting re-sharpening and PICC under load cycling. Model material parameters correspond here to medium-high strength steel. Notwithstanding, using common normalization techniques, generated solutions are applicable not to this sole material but to a similitude class fixed by the magnitudes of pertinent dimensionless parameters, such as the ratio of Young modulus $E$ to the yield stress $\sigma_{Y}$, Poisson coefficient $v$, and so on.

\section{MODELLING}

7 he results presented herein proceed from the extensive modeling of the mode I crack tip fields in elastoplastic material under SSY in terms of the crack tip autonomy dominated solely by the stress intensity factor (SIF) $K$ [36]. Model design (geometry, loading and constitutive material model) and the large-strain analysis procedure were basically the same as presented and substantiated elsewhere [14,18-20]. Their outline is as follows.

At large strains, material hardening approaches saturation, so that elastic perfectly-plastic constitutive model can be an acceptable approximation, provided the value of $\sigma_{Y}$ corresponds not to the initial yield point, but to some saturation stress level, the "effective" yield stress as modified by strain-hardening. Then, the model of ideal elastoplastic solid having $E=$ $200 \mathrm{GPa}, v=0.3$ and $\sigma_{Y}=600 \mathrm{MPa}$ with von Mises yield surface and associated flow rule was chosen. 
The simulations were performed for constant amplitude loadings at different SIF ranges $\Delta K=K_{\max }-K_{\min }$, and ratios $R=$ $K_{\min } / K_{\max }$, and the effect of a single overload was considered, too, along the following load cases:

(I) $\quad \Delta K=2 K_{0}, \mathrm{R}=0$,

(II) $\Delta K=K_{0}, R=0$,

(III) $\Delta K=K_{0}, \mathrm{R}=0.5$,

(IV) $\Delta K=K_{0}, R=0$, with an overload to $K_{\mathrm{ov}}=2 K_{0}$ at a single cycle of the loading path, where the reference value $K_{0}=30 \mathrm{MPa} \sqrt{\mathrm{m}}_{\mathrm{m}}$ corresponds to loading regimes of fatigue cracking in steels [2,34].

The model of undeformed crack was parallel-flanks slot of the width $b_{0}$ with semicircular tip, Fig. 1(a), as it has been repeatedly substantiated and used in analyses of cracks, e.g., by McMeeking [37], Needleman and Tvergaard [38], Rice $e t$ al. [39], or Toribio and Kharin [14,18-20]. The value of $b_{0}=5 \mu \mathrm{m}$ was taken as a reasonable choice for steels [40]. Model design was subdued to ensuring the SSY in terms of the $K$-dominated crack tip autonomy [36]. The choice was made $[19,20]$ in favor of full-scale specimens, which were double-edge-cracked panel (DECP) and central-cracked panel (CCP) under remote traction by uniform stress $\sigma_{\text {ap }}$, Fig. $1(b)$. Due to specimen symmetries, the boundary-value problems were stated for the same region $-a \leq X \leq W-a, 0 \leq Y \leq H$ (Fig. 1(b), shadowed), being $a$ the initial crack size, $2 W$ and $2 H$ the panel width and height, respectively. Boundary conditions were posed according to the specimen symmetries and loading, Fig. $1(c)$. To ensure the SSY, the model sizes were $a=15000 b_{0}, a / W=0.2$ and $W=H$. The SIF calibrations were specified using the specimen geometry factor according to the compendium [41] for DECP and CCP, respectively. The textbook $[34,36]$ estimation for the monotonic plastic zone dimension under plane strain, $R_{Y}=K^{2} /\left(3 \pi \sigma_{Y}^{2}\right)$, renders for the chosen material, geometry and loading $\max \left\{R_{Y} / a, R_{Y} / H, R_{Y} / W\right\} \leq 0.014<<1$, which ensures the SSY.

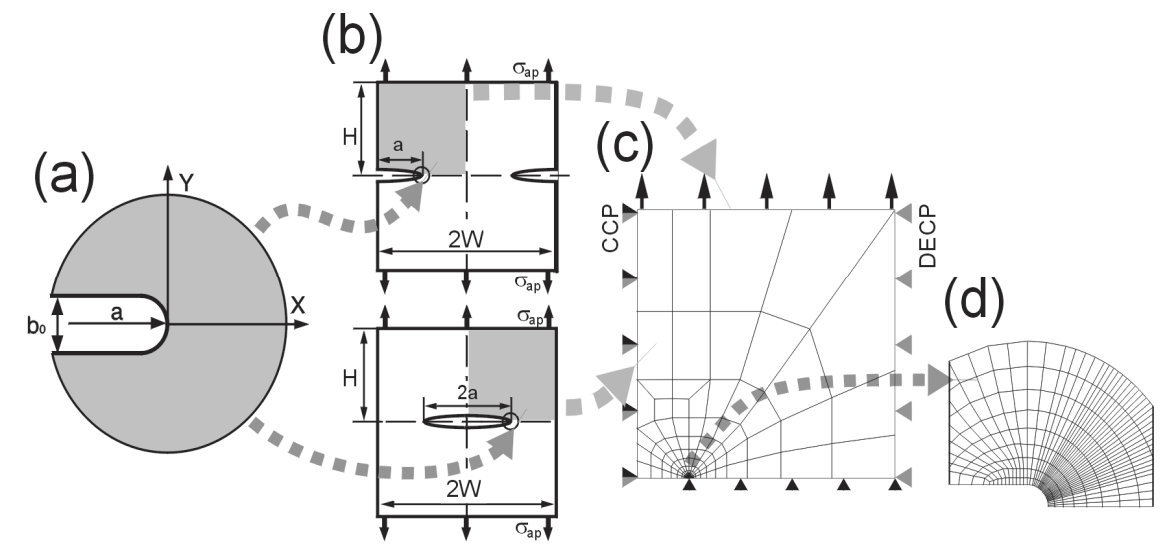

Figure 1: Model geometry and boundary conditions: $(a)$ crack, $(b)$ DECP and CCP specimens, $(c)$ global view of the FE model of the specimen quarters shadowed in $(b)$ with respective boundary conditions for CCP and DECP test-pieces, $(d)$ near tip mesh refinement.

Large-deformation elastoplastic solutions were generated using a finite element (FE) code with updated lagrangian formulation and additive decomposition of strain rates. The FE mesh design followed previous studies of large near tip deformations [37-39]. To overcome numerical problems associated with mesh degeneration at the crack tip, extensive trials were accomplished to ensure the solution mesh convergence for acceptable number of loading cycles. Re-meshing was considered to be not a suitable means to improve the FE model performance because of substantiated doubts [42] that, under the propensity to solution bifurcation of certain elastoplasticity problems due to their loss of ellipticity [43,44], small perturbations by re-meshing of the deformed tip shape in relation to the "true" one (yet unknown) can harm the results victim to pitfalls of a re-meshing technology. This later can break against mesh-sensitivity, which is rooted in the potential non-uniqueness of corresponding field solutions, to the extent that the relation between solution of the original continuum-mechanics problem and of its FE approximation may become questionable. To this end, it must not surprise to meet severe discrepancies between near tip deformations obtained using different mesh layouts, FE formulations and re-meshing procedures, which rendered crack faces folding without closure at the very first load cycles [17], as well as smooth surfaces arriving at closure at fairly large number of cycles [22, 23].

A variety of meshes permanently embedded in the material were explored attending the roles of FE size and shape, as well as of the interpolation order and integration scheme, as described elsewhere [42]. To postpone mesh degeneration, FE models of full-integration bi-linear quadrilateral elements were elaborated using rather high grade of element refinement 
and relying on suitable initial element shaping trying to ascertain the near tip deformation patterns yet to come in the deformed configuration in order to maintain the accumulated FE distortions within tolerable limits.

\section{RESULTS AND DISCUSSION}

G

enerated near tip solutions turned out to be fairly insensitive to the specimen geometry and loading in pertinent aspects. This way, the $K$-dominance over the crack tip zone was confirmed. For all geometry-and-loading cases, the crack-tip deformations evolved similarly to what is shown in Fig. 2. Plastic crack growth $\Delta a_{p}$ is there appreciated as an advancement of the tip apex $A_{0}$ deeper into material. This way, the Laird-Smith conceptual scheme, which is considered to be one of the intrinsic mechanisms of FCG in ductile materials [2,31-35], is visualized. Simulations evidence that the crack does grow under cyclic loading, although with no bond breaking.
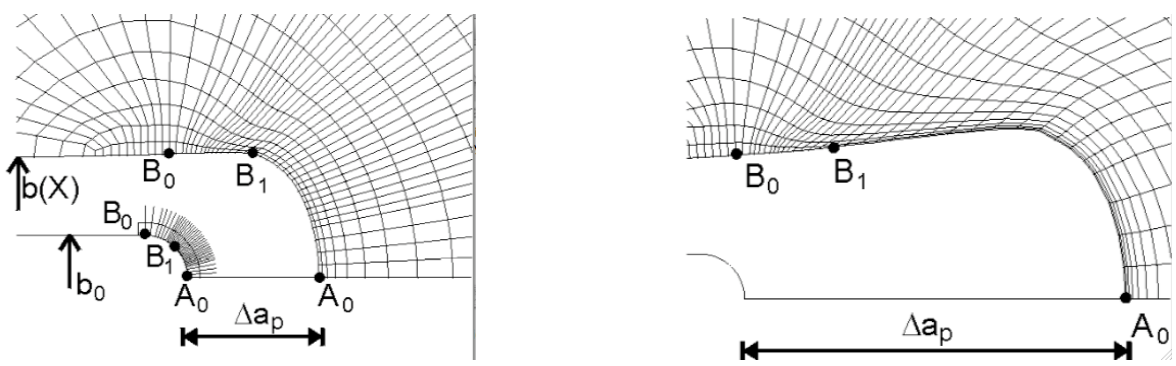

Figure 2: Crack tip deformations at unloading after accomplishing the first (a) and fifth (b) cycles of the load case I (DECP specimen); undeformed mesh fragment and tip contours are shown near the image center in $(a)$ and in the bottom-left corner in (b), respectively, together with the positions of material points $A_{0}, B_{0}$ and $B_{1}$ in the initial and deformed configurations.

The plastic crack advancements $\Delta a_{p} v$ s. cycle number $N$ are presented in Fig. 3. The slopes of these curves render corresponding rates of plastic crack growth $(d a / d N)_{p}$. Its acceleration with $\Delta K$ is there evident, whereas the effect of $R$ is rather vague. Calculated $(d a / d N)_{p}$ values are of the order of $10^{-6} \mathrm{~m} /$ cycle, which is proper for the Paris regime in steels [2,34]. The Paris-like equation $(d a / d N)_{p}=\mathrm{C} \Delta K^{\mathrm{m}}$ fits the numerical results at $m \approx 2.25$, which is reasonable for many alloys [34]. This mode of crack extension is sensible to an overload, which halts the crack advance (Fig. 3, load case IV). This way, the effects of $\Delta K$ and overload on FCG obtained by simulations agree with common experimental trends [2,34].

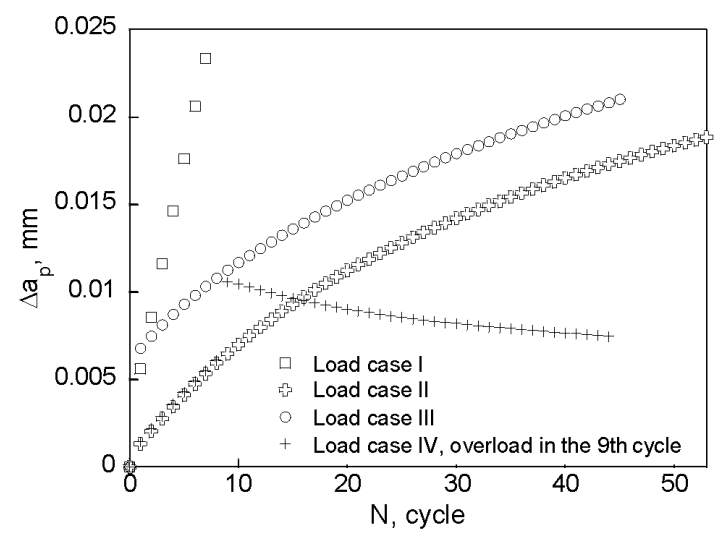

Figure 3: Crack growth $\Delta a_{p} v$ s. cycle number $N$ along indicated loading routes in CCP specimen (the points correspond to the crack tip positions at the load cycle ends).

No signs of PICC have ever occurred during simulated crack growth at $R \geq 0$. Although cracks upon unloading shrink in a wake behind the tip more than at the tip itself in all load cases (Fig. 2), deformed crack faces have never and nowhere got to the initial crack width, and so, no contact between crack surfaces could be approached. This holds for an appreciable crack advancement $\Delta a_{p}$ leaving a considerable plastic wake behind the tip, as it can be appreciated from Fig. 4 which displays crack penetration through substantial fraction of the plastic zones created in the first load cycle, the 
forward (monotonic) and the reverse ones, as well as the move forward of the actual cyclic plastic zone together with the crack tip, having these zones determined according to the criterion of non-zero equivalent plastic strain rate [20].

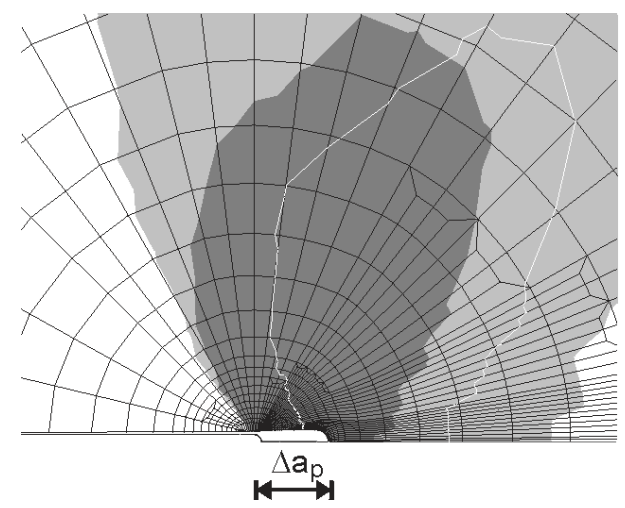

Figure 4: Plastic zones near the tip of the grown crack in the deformed solid configuration (meshed area) after accomplishing of 90 loading cycles along the route II: actual cyclic (reversed) plastic zone at the end of the 90th cycle (white contour line) and the material which was involved in plastic flow in the first loading cycle at its top (monotonic plastic zone, light-grey area) and bottom (reversed plastic zone at unloading, dark-grey area). Undeformed crack contour is present at the figure bottom.

Although presented modeling agrees with experimental trends of FCG, crack advancement by blunting re-sharpening can hardly be the entire mechanism of every FCG occurrence, as far as fatigue cracks usually evidence, e.g., by means of fractography, the signs of material damage and fracture. This may be the reason why simulated solely plastic crack growth is insensitive to the load ratio or manifests negative crack growth rate after an overload (Fig. 3). FCG is seen [2,4,34] to proceed usually by various mechanisms of deformation, damage and bond breaking which go on synergistically. As well, crack closure upon unloading can arise owing to a series of causes, both intrinsic (such as fracture surface roughness originated from various microstructural damage and fracture events, or stress-induced phase transformations, etc.) and extrinsic (e.g., in-crack debris, oxides, and so long) ones. However, presented results imply that crack closure can be neither a necessary requisite nor a decisive factor for the mentioned FCG trends.

Indeed, the plastic advancement of a crack behaves per se in agreement with well known experimental FCG trends, but with no signs of PICC. This latter may happen at larger number of cycles, as it occurred in simulations by Tvergaard [22,23], as well as may not. The supposed consequences of closure in FCG (e.g., the overload retardation or arrest [34]) do appear with no closure. Accordingly, the practiced indirect assessments of PICC-related issues from FCG behaviour [7] acquire a deal of spuriousness, which can make them artifacts.

Displayed results fairly agree in all pertinent aspects with other large-strain analyses of cracks under cyclic loading [22,23], but challenge those small- and finite-strain simulations (such as, e.g., [13,16,21,24]), where crack growth was imposed by means of cutting the material arbitrarily with a "virtual knife" in analyst's hands counting on neither the material nor the applied load. So, all these latter models were intrinsically unable to render per se any effect of the loading route on FCG, such as the influence of $\Delta K$ or overload. In contrast, presented modeling does this.

Moreover, by virtue of the permanent gluing of FE meshes to the material, it is revealed here how the crack grows by plastic straining, which renders material transfer from the crack front onto its lateral surfaces, as it does a neighborhood of the material point $B_{1}$ in Fig. 2. Material "bricks" situated initially a little bit aside the top $A_{0}$ of the arc $B_{0} B_{1} A_{0}$, which shapes the crack tip, move there sideways and build the crack flanks up, i.e., contribute to crack enlargement. This is lengthened additionally by stretching of these material "bricks", which are placed to form the crack flanks, Fig. 5(a), where large tensile plastic strain $\varepsilon_{y y}^{p}>0$ in the in-plane of the crack direction is observed, Fig. 5(b), which implies negative axial strain $\varepsilon_{Y Y} \approx-\varepsilon_{X X}<0$ near the crack tip by virtue of plane-strain large incompressible plasticity. This latter result agrees with experimental X-ray diffraction study of the strain fields near fatigue cracks, which reported the "negative anomaly" in $\varepsilon_{y y}$ strain behind the tip [45], but contradicts the results of small-strain numerical analysis [13] schematized in Fig. 5(c). This way, performed large-deformation simulations discard the mechanism of PICC implied from the small-strain modeling [13], where material elements behind the crack tip underwent out-of-plane plastic stretching $\varepsilon_{y y}^{p}>0$ accompanied by shrinking along the crack surfaces $\varepsilon_{x x}^{p} \approx-\varepsilon_{y y}^{p}<0$ by virtue of the plane-strain incompressible plasticity, Fig. 5(c). Accordingly, it was argued that this out-of-plane stretched material on the growing crack surfaces behind the tip could fill- 
in the crack, and this way enable the opposite crack surfaces to contact prematurely at unloading, i.e., produce PICC. Evidently, this contradicts to Figs. 2 and $5(a, b)$, where large displacements and rotations in the crack tip vicinity make material not to fill-in the crack behind the tip and render PICC, but to increment the crack flanks, i.e., they make the crack advance. This gives the reason to consider the suggested PICC origin as an artifact [13], as far as it appears to be not the naturally present feature of a crack under cyclic loading but a product of an analysis method.
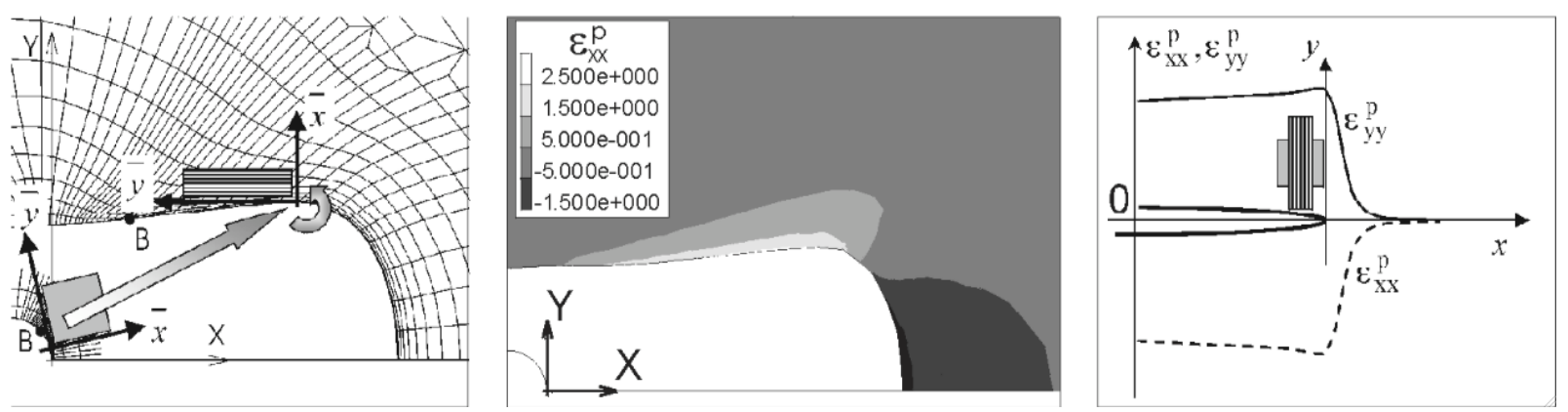

Figure 5: Deformations near the crack tip under cyclic loading: (a) crack-tip large-deformation patterns with a scheme showing how and where do material "bricks" with their glued local material frames $(\bar{x}, \bar{y})$ go by translation and rotation (arrows) from the initial configuration to make the crack grow; (b) contour bands of the plastic strain $\varepsilon_{X X}^{p}$ at the end of the sixth cycle of the route $\mathrm{I}$ in deformed solid configuration (undeformed crack tip is seen in the bottom-left corner) to illustrate the contribution of the "bricks" stretching to crack lengthening, but not to its closure; $(c)$ scheme of the small-deformation results [13, Fig. 8] used to substantiate the suggested origin of PICC in the supposed filling-in the crack with stretched material behind the tip; the squares shaded in gray and rectangles filled with line pattern represent in $(a)$ and $(c)$ the same material elements in the initial and deformed states, respectively.

\section{CONCLUSIONS}

he crack becomes larger after every cycle, i.e., it grows, as suggested the Laird-Smith concept, by means of plastic straining with certain rate $(d a / d N)_{p}$.

This growth reproduces the key experimental trends of FCG, such as the acceleration with $\Delta K$ and the hindering by overload, which have been repeatedly attributed to the crack closure.

Specimen compliance changes, which are frequently considered, following Elber [5], to be the evidences of crack closure, do occur in simulations.

Despite all that, no PICC has appeared, and revealed deformation patterns discard its supposed origination from filling-in the crack with plastically stretched material in the wake.

It does not matter now whether PICC could occur at substantially larger number of cycles or longer crack growth, or whether local material fracture through bond breaking is a necessary requisite for PICC to arise, but the point is that the supposed consequences of PICC do take place with no closure behind them, thereby bringing doubts about PICC.

\section{ACKNOWLEDGEMENTS}

7 he authors wish to acknowledge the financial support provided by the following Spanish Institutions: Ministry for Science and Technology (MCYT; Grant MAT2002-01831), Ministry for Education and Science (MEC; Grant BIA2005-08965), Ministry for Science and Innovation (MICINN; Grants BIA2008-06810, and BIA2011-27870) and Junta de Castilla y León (JCyL; Grants SA067A05, SA111A07, and SA039A08).

\section{REFERENCES}

[1] Overview. Advances in fatigue crack closure measurement and analysis, ASTM STP 1343, R.C. McClung, J.C. Newman, Eds., ASTM International, West Gonshohocken, (1999) XI.

[2] Ritchie, R. O., Mechanisms of crack propagation in ductile and brittle solids, Int. J. of Fract., 100 (1999) 55-83. 
[3] Louat, N., Sadananda, K., Duesbery, M., Vasudevan, A. K., A theoretical evaluation of crack closure, Met. Trans., A24 (1993) 2225-2232.

[4] Vasudevan, A. K., Sadananda, K., Glinka, G., Critical parameters for fatigue damage, Int. J. of Fatigue, 23 (2001) S39S53.

[5] Elber, W., Fatigue crack growth under cyclic tension, Eng. Fract. Mech., 2 (1970) 37-45.

[6] Macha, D. E., Corbly, D. M., Jones, J. W., On the variation of fatigue-crack-opening load with measurement location, Exp. Mech., 19 (1979) 207-213.

[7] Xu Yigeng, Gregson, P. J., Sinclair, I., Systematic assessment of compliance-based crack closure measurements in fatigue, Mater. Sci. and Eng., A284 (2000) 114-125.

[8] Deshpande, V. S., Needleman, A., van der Giessen, E., A discrete dislocation analysis of near-threshold fatigue crack growth, Acta Mater., 49 (2001) 3189-3203.

[9] Pippan, R., Riemelmoser, F. O., Visualization of the plasticity-induced crack closure under plane strain conditions, Eng. Fract. Mech., 60 (1998) 315-322.

[10] Bjerkén, C., Melin, S., Growth of a short fatigue crack - A long term simulation using a dislocation technique, Int. J. of Solids and Struct., 46 (2009) 1196-1204.

[11] Budiansky, B., Hutchinson, J. W., Analysis of closure in fatigue crack growth, J. of Appl. Mech., 45 (1978) $267-276$.

[12] Noroozi, A. H., Glinka, G., Lambert, S., Prediction of fatigue crack growth under constant amplitude loading and a single overload based on elasto-plastic crack tip stresses and strains, Eng. Fract. Mech., 75 (2008) 188-206.

[13] McClung, R. C., Thacker, B. H., Roy, S., Finite element visualisation of fatigue crack closure in plane stress and plane strain, Int. J. of Fract., 50 (1991) 27-49.

[14] Toribio, J., Kharin, V., Large crack-tip deformations and plastic crack advance during fatigue, Mater. Lett., 61 (2007) 964-967.

[15] Ellyin, F., Wu, J., Elastic-plastic analysis of a stationary crack under cyclic loading and effect of overload, Int. J. of Fract., 56 (1992) 189-208.

[16] Wu, J., Ellyin, F., A study of fatigue crack closure by elastic-plastic finite element for constant-amplitude loading, Int. J. of Fract., 82 (1996) 43-65.

[17] Levkovitch, V., Sievert, R., Svendsen, B., Simulation of fatigue crack propagation in ductile metals by blunting and resharpening, Int. J. of Fract., 136 (2005) 207-220.

[18] Toribio, J., Kharin, V., High-resolution numerical modelling of stress-strain fields in the vicinity of a crack tip subjected to fatigue, Fracture from Defects, EMAS, (1998) 1059-1064.

[19] Toribio, J., Kharin, V., Role of fatigue crack closure stresses in hydrogen assisted cracking, Advances in Fatigue Crack Closure Measurement and Analysis, ASTM STP 1343, R.C. McClung, J.C. Newman, Eds., ASTM International, West Gonshohocken, (1999) 440.

[20] Toribio, J., Kharin, V., Finite deformation analysis of the crack-tip fields under cyclic loading, Int. J. of Solids and Struct., 46 (2009) 1937-1952.

[21] Roychowdhury, S., Dodds, R. H., A numerical investigation of 3-D small-scale yielding fatigue crack growth, Eng. Fract. Mech., 70 (2003) 2363-2383.

[22] Tvergaard, V., On fatigue crack growth in ductile materials by crack-tip blunting, J. Mech. and Phys. of Solids, Vol. 52, 2004, pp. 2149-2166.

[23] Tvergaard, V., Overload effects in fatigue crack growth by crack-tip blunting, Int. J. of Fatigue, 27 (2005) $1389-1397$.

[24] Lei, Y., Finite Element crack closure analysis of a compact tension specimen, Int. J. of Fatigue, 30 (2008) 21-31.

[25] Lynn, A. K., DuQuesnay, D. L., Computer simulation of variable amplitude fatigue crack initiation behaviour using a new strain-based cumulative damage model, Int. J. of Fatigue, 24 (2002) 977-986.

[26] Nguyen, O., Repetto, E., Ortiz, M., Radovitzky, R., A cohesive model of fatigue crack growth, Int. J. of Fract., 110 (2001) 351-369.

[27] Chalant, G., Remy, L., Model of fatigue crack propagation by damage accumulation at the crack tip, Eng. Fract. Mech., 18 (1983) 939-952.

[28] Chan, K. S., Lankford, J., A crack tip strain model for the growth of small fatigue cracks, Scripta Met., 17 (1983) 529532.

[29] Fan, F., Kalnaus, S., Jiang, Y., Modelling of fatigue crack growth of stainless steel 304L, Mech. of Mater., 40 (2008) 961-973.

[30] Hurtley, P. J., Evans, W. J., A new method for predicting fatigue crack propagation rates, Mater. Sci. and Eng., A466 (2007) 265-273.

[31] Laird, C., Smith, G. C., Crack propagation in high stress fatigue, Phil. Mag., 8 (1962) 847-857. 
[32] Pelloux, R. M. N., Crack extension by alternating shear, Eng. Fract. Mech., 1 (1970) 697-704.

[33] Neumann, P., New experiments concerning the slip process at propagating fatigue cracks, Acta Met., 22 (1974) 11551165.

[34] Suresh S., Fatigue of materials, Cambridge University Press, Cambridge, (1991) 617.

[35] Riemelmoser, F. O., Pippan, R., Stüwe, H. P., An argument for a cycle-by-cycle propagation of fatigue cracks at small stress intensity ranges, Acta Mater., 46 (1998) 1793-1799.

[36] Kanninen, M.F., Popelar, C.H., Advanced fracture mechanics, Oxford University Press, New York, (1985) 562.

[37] McMeeking, R. M., Finite deformation analysis of crack tip opening in elastic-plastic materials and implications for fracture, J. Mech. and Phys. of Solids, 25 (1977) 357-381.

[38] Needleman, A., Tvergaard, V., Crack-tip stress and deformation fields in a solid with vertex on its yield surface, Elastic-Plastic Fracture: Second Symposium - Inelastic Crack Analysis, ASTM STP 803, C.F. Shih, J.P. Gudas, Eds., ASTM International, 1 (1983) 80.

[39] Rice, J. R., McMeeking, R. M, Parks, D. M., Sorensen, E. P., Recent finite element studies in plasticity and fracture mechanics, Comput. Meth. Applied Mech. and Eng., 17/18 (1979) 411-442.

[40] Handerhan, K. J., Garrison, W. M., Jr., A study of crack tip blunting and the influence of blunting behavior on the fracture toughness of ultra high strength steels, Acta Met. et Mater., 40 (1992) 1337-1355.

[41] Savruk, M. P., Stress intensity factors in solids with cracks, Naukova Dumka, Kiev, (1988) 619

[42] Toribio, J., Kharin, V., Comments on simulations of fatigue crack propagation by blunting and re-sharpening: the mesh sensitivity, Int. J. of Fract., 140 (2006) 285-292.

[43] Hill, R., Acceleration waves in solids, J. Mech. and Phys. of Solids, 10 (1962) 1-16.

[44] Rice, J. R., The localization of plastic deformation, Theoretical and Applied Mechanics, North-Holland, Amsterdam, (1977) 207-220.

[45] Croft, M., Zhong, Z., Jisrawi, N., Zakharchenko, I., Holtz, R.L., Skaritka, J., Fast, T., Sadananda, K., Lakshmipathy, M., Tsakalakos, T., Strain profiling of fatigue crack overload effects using energy dispersive X-ray diffraction, Int. J. of Fatigue, 27 (2005) 1408-1419. 